
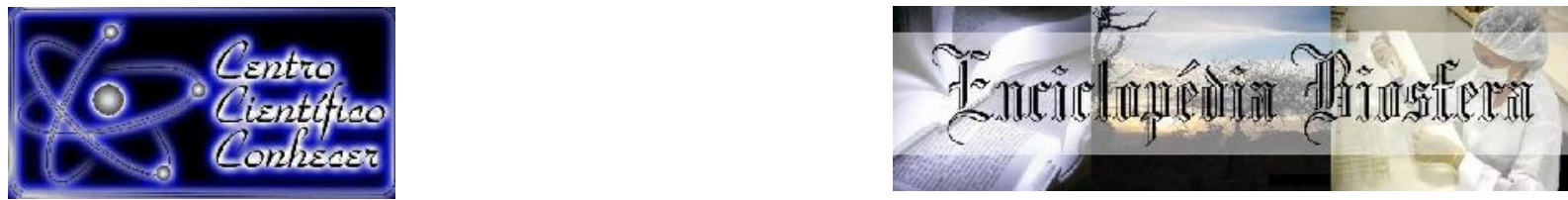

\title{
MODELOS DE RELAÇÃO HIPSOMÉTRICA POR CLASSE DE APROVEITAMENTO EM FLORESTA DE TRANSIÇÃO CERRADO-AMAZÔNIA NO MATO GROSSO
}

\footnotetext{
Rafaella De Angeli Curto ${ }^{1}$, Charlote Wink ${ }^{1}$, Emanuel José Gomes de Araújo ${ }^{2}$, Sintia Valerio Kohler ${ }^{3}$

${ }^{1}$ Professora, Doutora, Instituto de Ciências Agrárias e Ambientais, Universidade Federal de Mato Grosso, Campus Sinop, Sinop, MT, Brasil.

rafaellacurto@yahoo.com.br

2 Professor, Doutor, Departamento de Silvicultura, Instituto de Florestas, Universidade Federal Rural do Rio de Janeiro, Seropédica, RJ, Brasil.

${ }^{3}$ Professora, Doutora, Departamento de Engenharia Florestal, Universidade Estadual do Centro-Oeste, Campus Irati, Irati, PR, Brasil.
}

Recebido em: 22/09/2018 - Aprovado em: 23/11/2018 - Publicado em: 03/12/2018 DOI: 10.18677/EnciBio_2018B60

\begin{abstract}
RESUMO
Em vista das normas dos Planos de Manejo Florestal Sustentável, este trabalho teve como objetivo verificar a identidade de modelos de relação hipsométrica em diferentes classes de aproveitamento em fitofisionomia de Floresta de Transição CerradoAmazônia. O estudo foi realizado no Parque Florestal de Sinop, em Sinop, Mato Grosso. Avaliou-se aleatoriamente o diâmetro a altura do peito e a altura total de 96 árvores distribuídas nas classes de regeneração $(10$ a 29,9 cm), remanescentes $(30$ a $49,9 \mathrm{~cm})$ e de corte $(>50 \mathrm{~cm})$. Posteriormente realizou-se 0 ajuste e avaliação qualitativa de modelos de relação hipsométrica, por meio de diferentes estatísticas. As equações hipsométricas estratificadas por classe de aproveitamento resultam em aumento da precisão quando trata-se de árvores nas classes de regeneração (10 a $29,9 \mathrm{~cm}$ de DAP) e de corte (> $50 \mathrm{~cm}$ ). Quanto a identidade de modelos, é possível o agrupamento de árvores nas classes regeneração com as remanescentes, e agrupamento de árvores remanescentes com árvores da classe de corte, para realização simultânea dos ajustes. Pela estratificação e aumento de precisão, pode-se reduzir o tempo de coleta de dados e os custos envolvidos na atividade, com a redução da intensidade amostral.
\end{abstract}

PALAVRAS-CHAVE: altura, custo, identidade de modelos.

\section{DIAMETER-HEIGHT MODELS BY CLASS OF ADAPTATION IN AMAZONIA- CERRADO TRANSITION FOREST}

\begin{abstract}
In view of the norms of the Plans of Sustainable managcmcnt, the objective was to verify the identity of models of diameter-height relation in different classes of use in
\end{abstract}


phytophysiognomy of Amazonia-Cerrado transition forest. The study was carried out in the Sinop Forest Park, in Sinop, Mato Grosso. Were randomly evaluated the diameter at breast height and the total height of 96 trees were distributed in classes: regeneration $(10$ to $29.9 \mathrm{~cm})$, remaining $(30$ to $49.9 \mathrm{~cm})$ and cut $(>50 \mathrm{~cm})$. Subsequently, the fit and qualitative evaluation of diameter-height models were carried out, through different statistics. The diameter-height equations stratified by performance class result in increased accuracy when dealing with trees in the regeneration classes (10 to $29.9 \mathrm{~cm}$ of DAP) and cut $(>50 \mathrm{~cm})$. As for the identity of models, it is possible to agroup trees in the regeneration classes with the remaining ones, and grouping of remaining trees with trees of the cut class, for simultaneous realization of the fits. With the stratification and the increase of precision, the data collection time and the costs involved in the activity can be reduced, reducing the sample intensity.

KEYWORDS: Cost, height, identity of models.

\section{INTRODUÇÃO}

Em Planos de Manejo Florestal Sustentável (PMFS) é de primordial interesse que se possa quantificar fidedignamente o estoque de madeira na floresta, fornecendo subsídio essencial à tomada de decisões, garantindo a conservação e perpetuidade para as futuras gerações. Simultaneamente, os órgãos ambientais competentes, por meio de suas atribuições legais, visam ao viável desenvolvimento de florestas inequiâneas submetidas a PMFS, fazendo-se necessário a fiscalização e controle do estoque correto de madeira proveniente dessas áreas, sendo exigido estimativas de volume mais próximas possíveis do real (GAMA et al.,2017).

Assim, conforme estabelecido pelo Artigo 10 da Resolução Conama no 406 de 02/02/2009 e pela Secretaria de Estado de Meio Ambiente de Mato Grosso (SEMA-MT) pelo Decreto № 1.862 de 24/03/2009, todos os PMFS aprovados no bioma Amazônia devem apresentar equações de volume desenvolvidas especificamente para a área manejada, visando obter o volume comercial das árvores em pé, a partir do segundo Plano Operacional Anual (POA). No primeiro POA, a legislação estabelece fator de forma padrão igual a 0,7 para todas as espécies (GAMA et al., 2017).

A importância em obter equações de volume específicas para cada área, foi abordada por GAMA et al. (2017), a fim de não quantificar equivocadamente os estoques volumétricos, uma vez que isso ocasiona prejuízos econômicos, comprometendo o planejamento. Deste modo, a identificação de técnicas que possibilitem a mensuração de variáveis dendrométricas para a obtenção do volume com minimização de erros, é de extrema importância. Para isso, se busca a mensuração de dimensões básicas como diâmetro a altura do peito (DAP) e a altura de árvores (FELICIANO et al., 2016), havendo uma constante busca de alternativas visando otimizar as suas formas de obtenção.

A SEMA-MT por meio da Instrução Normativa nº 005 de 02/07/2014, que estabelece roteiros específicos para PMFS Madeireiro, prevê a possibilidade de apresentação do fator de forma desenvolvido especificamente para a área manejada, a partir do segundo POA, o que ressalta a importância da obtenção da altura com precisão. Com os dados de altura das árvores, é possível obter o volume de madeira de uma floresta e assim, planejar corretamente retiradas de madeira, além de, segundo SOUZA ; SOUZA (2004), ser possível compreender a estrutura vertical de uma 
comunidade, por permitir estimar as espécies que apresentam maior importância ecológica, gerando importante significado para fins de manejo.

Porém, a mensuração da altura de árvores é uma atividade onerosa devido as dificuldades atribuídas às condições do ambiente (SILVA et al., 2017), pelo alto custo, devido ao tempo demandado para a atividade e em alguns casos, devido a limitação por falta de recursos para a aquisição de instrumentos de medição que sejam precisos e acurados. Por isso, é comum o uso da forma expedita, em que o operador obtém estimativas sem nenhum instrumento, apoiando-se apenas em sua habilidade pessoal (CURTO et al., 2013). No entanto, tal atividade pode resultar em estimativas tendenciosas se o estimador não possuir adequado treinamento (CURTO et al., 2018), apesar dessa forma de estimação da altura ser permitida na execução de PFMS.

Desta forma, o desenvolvimento de métodos, visando a estimativa da altura, com menor demanda de tempo, mantendo uma boa precisão e eliminando possíveis erros causados pelo mensurador, são de grande interesse na área florestal (LINGNAU et al., 2008), podendo-se assim, empregar estimativas por meio do desenvolvimento de relações hipsométricas.

Diferente das relações hipsométricas usualmente utilizadas em florestas equiâneas, as relações hipsométricas em florestas inequiâneas tendem a apresentar baixa precisão devido a elevada heterogeneidade dessas florestas (CURTO et al., 2014). Isso ocorre, pois, as relações hipsométricas são afetadas por diferentes aspectos, como a espécie, a posição sociológica, a idade, o tamanho da copa, a densidade, dentre outros (MACHADO et al., 2008).

Assim, para não cometer erros grosseiros de estimação da altura, e consequentemente do volume, alguns cuidados devem ser tomados, como atentar-se a composição da amostra utilizada no ajuste da relação hipsométrica. De acordo com Curto et al. (2014), uma estratégia importante seria a estratificação dos dados em grupos com características semelhantes, visando aumento na precisão do ajuste dessas relações.

Portanto, tendo-se em vista a possibilidade do uso de equações hipsométricas visando a redução de custos das atividades de mensuração florestal, objetivou-se com o presente estudo verificar a identidade de modelos para relação hipsométrica de árvores em diferentes classes de aproveitamento, em fitofisionomia de Floresta de Transição Cerrado-Amazônia, observando as normas para Planos de Manejo Florestal Sustentável.

\section{MATERIAL E MÉTODOS}

\section{Área de estudo}

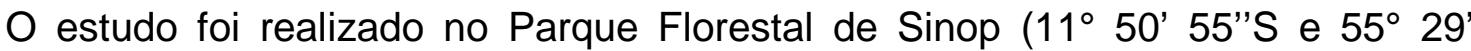
55"O), no município de Sinop, no Mato Grosso. A área possui 103,98 hectares e conforme sua composição florística e estrutura, é caracterizada segundo Araujo et al. (2009), como área de transição Cerrado-Floresta Amazônica. A área escolhida para abordagem do estudo representa fidedignamente as características de composição e estrutura de áreas em transição da vegetação de Cerrado e Amazônia, além de que, o município de Sinop está inserido em uma região com grande número de aprovações de PMFS. 
Segundo a classificação de Köppen e de Thornthwaite a região é do tipo climático Aw e B2wA'a, caracterizado pela presença de estações chuvosa (entre outubro e abril) e seca (de maio a setembro) bem definidas, com precipitação total anual variando de aproximadamente 1300 a $2000 \mathrm{~mm}$, com temperatura média mensal de $23,5^{\circ} \mathrm{C}$ a $25,5^{\circ} \mathrm{C}$ (MOTA et al., 2013).

\section{Coleta de dados}

Foram selecionadas aletoriamente 96 árvores, distribuídas igualmente em três classes de diâmetro a altura do peito (1,30 m do solo), variando entre 10 e $29,9 \mathrm{~cm}$, consideradas como regeneração (Classe 1), entre 30 e $49,9 \mathrm{~cm}$, consideradas árvores remanescentes (Classe 2) e maiores que $50 \mathrm{~cm}$ de DAP (Classe 3), destinadas a corte. Tal estratificação foi adotada levando-se em conta as classes de aproveitamento conforme a Instrução Normativa no 5, de 11/12/2006 do Ministério do Meio Ambiente e Decreto no 1.862 de 24/03/2009 da Secretaria de Estado de Meio Ambiente de Mato Grosso (SEMA-MT).

Após a seleção dos indivíduos nas respectivas classes de aproveitamento, os mesmos foram mensurados quanto à sua altura total, utilizando o hipsômetro Vertex IV®, com devida comprovação de sua exatidão, conforme Silva et al. (2012), Curto et al. (2013), Villasante e Fernandez (2014), Feliciano et al. (2016), e devido principalmente a inviabilidade para a obtenção da altura de forma direta, com escalada ou derrubada.

\section{Ajustes de equações hipsométricas}

Para o conjunto de dados, foram ajustados dez modelos hipsométricos (Tabela 1) utilizando o Microsoft Excelß. As significâncias dos coeficientes foram avaliadas pelo teste $t$, a $5 \%$ de probabilidade.

TABELA 1 - Modelos de relação hipsométrica ajustados para o conjunto de dados.

\begin{tabular}{ccc}
\hline Número & Autores & Modelos hipsométricos \\
\hline 1 & Linear simples & $h=\beta_{0}+\beta_{1} D A P+\varepsilon$ \\
2 & Trorey & $h=\beta_{0}+\beta_{1} D A P+\beta_{2} D A P^{2}+\varepsilon$ \\
3 & Assmann & $h=\beta_{0}+\beta_{1} / D A P+\varepsilon$ \\
4 & Henricksen & $h=\beta_{0}+\beta_{1} L n(D A P)+\varepsilon$ \\
5 & Stoffels & $\operatorname{Ln}(h)=\beta_{0}+\beta_{1} L n(D A P)+\varepsilon$ \\
6 & Curtis & $\operatorname{Ln}(h)=\beta_{0}+\beta_{1} / D A P+\varepsilon$ \\
7 & Petterson & $h=\left[1 /\left(\beta_{0}+\beta_{1} / D A P\right)\right]^{3}+1,3+\varepsilon$ \\
8 & Naslund (Prodan) & $h=\frac{D A P^{2}}{\beta_{0}+\beta_{1} D A P+\beta_{2} D A P^{2}}+\varepsilon$ \\
9 & Naslund & $h=\left(\frac{D A P^{2}}{\beta_{0}+\beta_{1} D A P^{2}}+1,30\right)+\varepsilon$ \\
10 & Naslund (Prodan) & $h=\left(\frac{D A P^{2}}{\beta_{0}+\beta_{1} D A P+\beta_{2} D A P^{2}}+1,30\right)+\varepsilon$
\end{tabular}

Em que: $D A P=$ diâmetro a $1,30 \mathrm{~m}$ de altura do solo $(\mathrm{cm}) ; h=$ altura total $(\mathrm{m}) ; \ln =$ logaritmo neperiano; $\beta_{i}$ = parâmetros do modelo ajustado $(i=0,1,2 \ldots n)$ e; $\varepsilon=$ erro de estimativa. 


\section{Avaliação e escolha dos modelos}

A escolha da melhor equação hipsométrica ajustada, para o conjunto dos dados, foi realizada com base nos critérios do erro padrão da estimativa em percentagem ( $S_{\mathrm{yx}}$ $\%)$ e do coeficiente de determinação ajustado em percentagem ( $\left.R^{2} a j \%\right)$. Nos casos de equações logaritmizadas, visto que essas equações tendem a subestimar os valores da altura, utilizou-se um fator de conversão, visando corrigir esse erro, por meio da multiplicação das alturas estimadas pelo Fator de Meyer (FM) para recalcular o erro padrão da estimativa, conforme descrito por Machado et al. (2015). Já o índice de ajuste de Schulaegel (IA) foi utilizado visando permitir a comparação com o coeficiente de determinação ajustado $\left(R^{2} a j \%\right)$ das equações de diferentes naturezas (MACHADO et al., 2015).

Para verificar a ocorrência ou não de tendências nas estimativas da variável altura, foram realizadas análises gráficas de resíduos em função do DAP $(\mathrm{cm})$ observado, bem como testes complementares (Tabela 2) por meio das estatísticas do Viés (V), Média das Diferenças Absolutas (MD) e Desvio Padrão das Diferenças (DPD), conforme descrito por Figueiredo Filho et al. (1996).

Após a seleção da melhor equação para o conjunto dos dados, o modelo correspondente foi utilizado para o ajuste com os dados estratificados de acordo com as classes de aproveitamento pré-estabelecidas. A avaliação das equações por classe de aproveitamento foi realizada por meio das estatísticas de precisão já descritas anteriormente.

TABELA 2 - Critérios e estimadores de avaliação dos modelos utilizados para a estimativa da altura total.

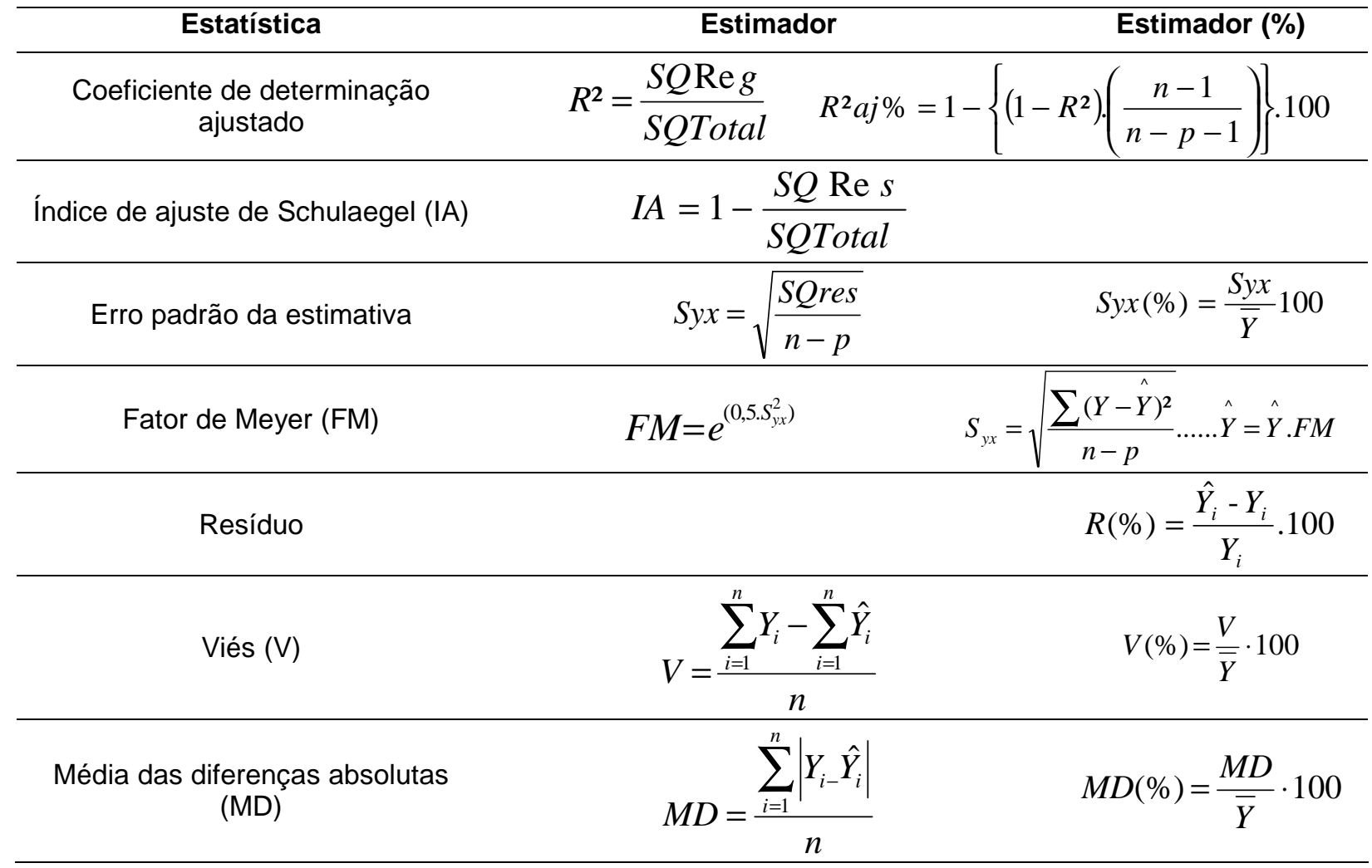


Desvio padrão das diferenças

(DPD)

$$
D P D=\sqrt{\frac{\left(\sum_{i=1}^{n} d i^{2}-\left(\frac{\sum_{i=1}^{n} d i}{n}\right)^{2}\right)}{n-1}}
$$

$$
D P D(\%)=\frac{D P D}{\bar{Y}} \cdot 100
$$

Em que: SQReg = soma dos quadrados da regressão; SQTotal = soma dos quadrados do total; SQRes = soma dos quadrados dos resíduos; $n=$ número de observações; $p=$ número de coeficientes de cada modelo utilizado; $Y_{i}=$ Altura da i-ésima árvore medida com o hipsômetro Vertex®, $(\mathrm{m}) ; \widehat{Y}_{l}=$ altura da iésima árvore obtida com a equação ajustada $(\mathrm{m}) ; \bar{Y}=$ média das alturas observadas $(\mathrm{m}) ; d i=(Y i-\hat{Y i})$.

\section{Identidade de modelos}

Após o ajuste das equações de relação hipsométrica para as diferentes classes de aproveitamento pré-estabelecidas, buscou-se identificar a igualdade estatística entre as equações obtidas, por meio do teste de identidade de modelos de regressão linear, desenvolvido por Graybill (1976) e descrito por Regazzi (1996).

A técnica consiste em verificar se existe semelhança estatística entre equações lineares provenientes do mesmo modelo, por meio do teste F de Graybill (Tabela 3), em que se emprega a diferença entre a soma de quadrados da regressão $\left(S Q_{\text {reg }}\right)$ dos modelos completos (ajustado por classe de aproveitamento) e o modelo reduzido (ajustado para todo o conjunto de dados). Verificadas diferenças significativas entre estas duas estruturas procede-se o mesmo procedimento de ajuste considerando todas as possíveis combinações do agrupamento das classes.

TABELA 3 - Análise de variância do teste de identidade de modelos lineares.

\begin{tabular}{lcccc}
\hline Fonte de Variação & $\mathbf{G L}$ & $\mathbf{S Q}$ & $\mathbf{Q M}$ & $\mathbf{F}$ \\
\hline Modelo Completo & $(\mathrm{hp})$ & $\mathrm{SQ}_{\text {reg-completo }}=\mathrm{SQ}_{1}+\mathrm{SQ}_{2}+\ldots$ & & \\
Modelo Reduzido & $\mathrm{p}$ & sQreg-reduzido & & \\
Diferença & $(\mathrm{h}-1) \mathrm{p}$ & $\mathrm{SQ}_{\text {reg-completo }}-\mathrm{SQ}_{\text {reg-reduzido }}$ & $\mathrm{SQ}_{\text {diferença }} /(\mathrm{h}-1) \mathrm{p}$ & $\mathrm{QM}_{\text {resíduo }}$ \\
Resíduo & $\mathrm{n}-(\mathrm{hp})$ & $\mathrm{SQ}_{\text {total }}-\mathrm{SQ}_{\text {reg-completo }}$ & $\mathrm{SQ}_{\text {resíduo }} /(\mathrm{n}$ - $\mathrm{hp})$ & \\
\hline Total & $\mathrm{n}$ & $\mathrm{SQ}_{\text {(total) }}$ & & \\
\hline
\end{tabular}

Em que: $\mathrm{GL}$ = grau de liberdade; $\mathrm{SQ}$ = soma de quadrados; $\mathrm{SQ}_{\mathrm{reg}}=$ soma de quadrados da regressão; $\mathrm{QM}=$ quadrado médio; $\mathrm{h}$ = número de tratamentos agrupados; $\mathrm{p}=$ número de parâmetros do modelo; $\mathrm{n}=$ número de observações do modelo completo.

Fonte: Regazzi (1996).

O teste de identidade de modelos desenvolvido por Graybill (1976) tem o intuito de verificar a igualdade de um conjunto de equações de regressão, testando a hipótese de que as equações completas são idênticas a equação reduzida $\left(\mathrm{H}_{0}\right)$ ou de que nem todas as equações completas são idênticas a equação reduzida $\left(\mathrm{H}_{1}\right)$. Deste modo, a não rejeição de $\mathrm{H}_{0}$ permite concluir a um nível de significância, nesse caso, de $5 \%$, que as " $n$ " equações não diferem estatisticamente entre si ( $F$ calculado < F tabelado), ou seja, as equações ajustadas com as estimativas dos parâmetros comuns podem ser usadas como uma estimativa das demais equações envolvidas. Do contrário, se $F$ calculado $\geq \mathrm{F}$ tabelado, se rejeita $\mathrm{H}_{0}$ e conclui-se que não é impossível o uso de um modelo único com parâmetros comuns. 


\section{RESULTADOS E DISCUSSÃO}

Para a estimativa da altura total sem a estratificação dos dados, os parâmetros para as equações foram significativos com $95 \%$ de probabilidade pelo teste $t$ para a maioria dos modelos ajustados (TABELA 4), exceto para os modelos de Naslund (8 e 9).

TABELA 4 - Equações ajustadas e estatísticas de precisão para o conjunto de dados obtidos em floresta de transição Cerrado e Amazônia, Sinop, MT.

\begin{tabular}{|c|c|c|c|c|c|c|c|c|}
\hline Modelo & $\widehat{\boldsymbol{\beta}_{0}}$ & $\widehat{\boldsymbol{\beta}}_{1}$ & $\widehat{\boldsymbol{\beta}_{2}}$ & $\mathbf{R}^{2}{ }_{\mathrm{aj}} \%$ & $S_{y x} \%$ & $\mathrm{~V} \%$ & MD\% & DPD\% \\
\hline Linear simples & $10,3534^{*}$ & $0,3111^{*}$ & & 68,38 & 17,33 & $1,12 \mathrm{E}-14$ & 12,67 & 17,24 \\
\hline Trorey & $6,4683^{*}$ & $0,5315^{*}$ & $-0,0025^{\star}$ & 70,42 & 16,88 & $3,05 \mathrm{E}-14$ & 11,74 & 16,70 \\
\hline Assmann & $32,3723^{*}$ & $-295,4491^{*}$ & & 62,89 & 18,78 & $-9,45 E-15$ & 14,31 & 18,68 \\
\hline Henricksen & $-17,4415^{\star}$ & $11,2782^{*}$ & & 70,37 & 16,78 & $1,63 \mathrm{E}-16$ & 11,96 & 16,69 \\
\hline Stoffels & $1,1638^{*}$ & $0,5367^{*}$ & & 70,29 & 15,73 & 1,18 & 11,82 & 16,67 \\
\hline Curtis & $3,5487^{*}$ & $-14,5043^{*}$ & & 67,07 & 16,83 & 1,59 & 12,97 & 17,52 \\
\hline Petterson & $0,3044^{*}$ & $1,9798^{*}$ & & 67,92 & 17,46 & 2,24 & 12,63 & 17,22 \\
\hline Naslund (Prodan) & $-10,2834^{\mathrm{ns}}$ & $1,5026^{*}$ & $0,0130^{\star}$ & 68,04 & 17,52 & 1,82 & 12,69 & 17,24 \\
\hline Naslund & $20,2167^{\star}$ & $0,0305^{\star}$ & & 64,44 & 18,38 & 3,46 & 13,97 & 17,95 \\
\hline Naslund (Prodan) & $-10,3476^{\text {ns }}$ & $1,6246^{*}$ & $0,0127^{\star}$ & 68,40 & 17,42 & 2,10 & 12,50 & 17,11 \\
\hline
\end{tabular}

De acordo com Levine et al. (2016), em modelos de regressão, cada parâmetro associado a variáveis independentes $\left(\beta_{1} \ldots \beta n\right)$, representam a inclinação da variável dependente $(Y)$ em relação à independente $(X)$ a ele relacionada, já o $\beta_{0}$ é a interseção de $\mathrm{Y}$, representando o seu valor médio quando as variáveis independentes $\left(\mathrm{X}_{\mathrm{i}}\right)$ são iguais a zero. Portanto, quando o parâmetro $\beta_{0}$ não é significativo não se tem prejuízos na estimativa da variável. Deste modo, qualquer um dos modelos ajustados poderia ser utilizado na estimativa da altura total de todas as árvores.

A partir da análise estatística dos modelos com parâmetros significativos, verificou-se erros inferiores a $20 \%$, no entanto, os dois melhores modelos ajustados foram os de Henricksen e Stoffels, de acordo com os menores erros observados, conjuntamente com os maiores coeficientes de determinação ajustados.

Em florestas inequiâneas, conforme relatado por Curto et al., (2014), existe uma grande variabilidade de alturas para uma mesma classe de diâmetro, o que resulta em uma relação hipsométrica não muito forte e, consequentemente, em coeficientes de determinação baixos e erros padrões de estimativa altos. Além disso, baixos valores de coeficiente de determinação segundo Araújo et al. (2012) estão associadas ao comportamento da distribuição diamétrica do tipo exponencial negativa (J invertido) em florestas naturais, o que é explicado por uma grande concentração de indivíduos nas classes inferiores de diâmetro e uma grande variabilidade de alturas. Deste modo, por se tratar de uma floresta inequiânea, os modelos ajustados no presente estudo são considerados satisfatórios para descrever a relação altura-diâmetro, uma vez que apresentam erros menores do que os observados por Curto et al. (2014) em Floresta Estacional Semidecidual no Espírito Santo, por Santos et al. (2016) no Cerrado Tocantinense e Staub et al. (2014) em plantio de restauração contendo 79 espécies nativas da Mata Atlântica, todos estimando a altura total. Tal erro se agrava ainda mais quando se trata de estimativa de altura comercial, visto que de acordo com Gimenez et 
al. (2015), para espécies comerciais madeireiras Amazônia a correlação entre as variáveis DAP e altura comercial é baixa $(0,22)$.

Para a interpretação das estatísticas Viés (V), Média das Diferenças Absolutas (MD) e Desvio Padrão das Diferenças (DPD) os menores valores indicam respectivamente menores tendências, menores amplitudes de erro e maior homogeneidade dos erros ao longo do eixo, demonstrando que o ajuste apresenta maior exatidão. Tais estatísticas reforçam o que se vê na análise gráfica dos resíduos (Figura 1), em que, com base no Viés (V), é possível observar que ambos os modelos escolhidos (Henricksen e Stoffels) apresentaram uma tendência quase nula em subestimar as alturas, isso porque, os valores positivos e negativos da estatística Viés indicam subestimativa e superestimativa, respectivamente. Portanto, nota-se que os modelos foram semelhantes quanto a precisão considerando o ajuste para o conjunto dos dados em todas as classes de aproveitamento avaliadas.
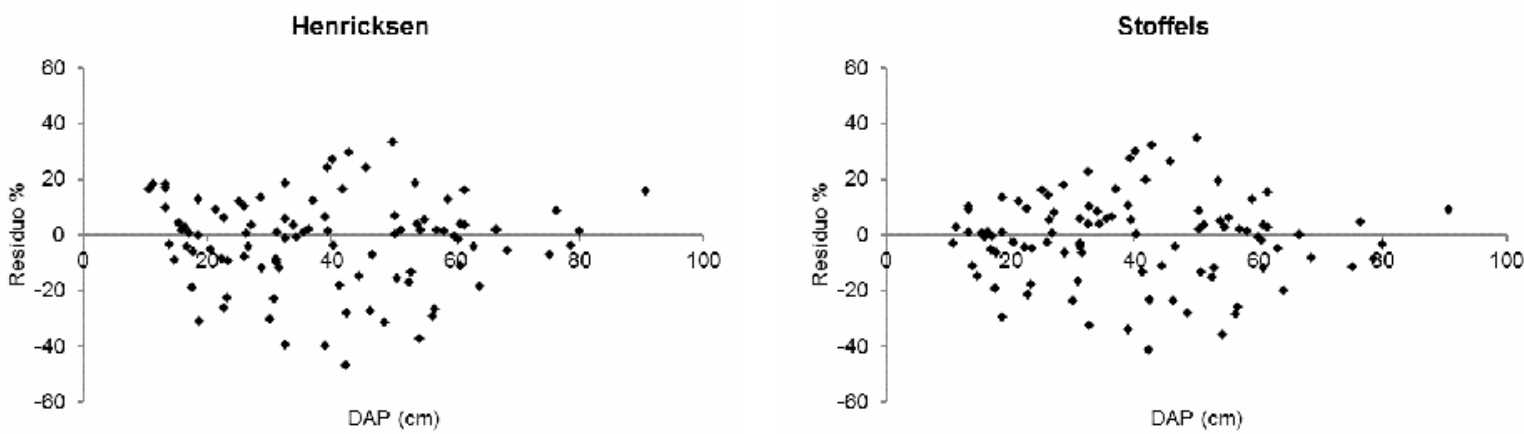

FIGURA 1 - Distribuição dos resíduos de altura, em percentagem, para os modelos de Henricksen e Stoffels, para árvores variando em todas as classes de diâmetro em floresta de transição Cerrado e Amazônia, Sinop, MT.

No ajuste das equações por classe de aproveitamento (Tabela 2), percebe-se que para as classes remanescentes e de corte foram verificadas a não significância do coeficiente $\beta_{0}$, porém, isso não causa prejuízo na estimativa da altura total.

TABELA 2 - Equações ajustadas e estatísticas de precisão para árvores nas classes de regeneração, remanescente e corte, em floresta de transição Cerrado e Amazônia, Sinop, MT.

\begin{tabular}{ccccccccc}
\hline & Modelo & $\widehat{\boldsymbol{\beta}}_{\mathbf{0}}$ & $\widehat{\boldsymbol{\beta}}_{\mathbf{1}}$ & $\mathbf{R}^{2}{ }_{\text {aj }}$ & $\mathbf{S}_{\mathrm{yx}} \%$ & $\mathbf{V} \%$ & $\mathbf{M D} \%$ & $\mathbf{D P D} \%$ \\
\hline Regeneração & Henricksen & $-12,5023^{*}$ & $9,6245^{*}$ & 67,17 & 11,85 & $3,15 \mathrm{E}-15$ & 9,24 & 11,65 \\
$(10$ a $29,9 \mathrm{~cm})$ & Stoffels & $0,9695^{*}$ & $0,6021^{*}$ & 69,40 & 11,41 & 0,63 & 8,73 & 11,23 \\
\hline Remanescente & Henricksen & $-43,5684^{\mathrm{ns}}$ & $18,5202^{*}$ & 22,22 & 22,54 & $2,7 \mathrm{E}-14$ & 17,23 & 22,18 \\
$(30$ a 49,9 cm $)$ & Stoffels & $0,5460^{\mathrm{ns}}$ & $0,7135^{*}$ & 20,99 & 22,59 & 2,20 & 17,44 & 22,24 \\
\hline Corte & Henricksen & $-41,0044^{*}$ & $16,9623^{*}$ & 37,33 & 11,68 & $-8,8 \mathrm{E}-14$ & 9,14 & 11,49 \\
$(\geq 50 \mathrm{~cm})$ & Stoffels & $0,9766^{\mathrm{ns}}$ & $0,5767^{*}$ & 37,94 & 11,60 & 0,66 & 9,21 & 11,41 \\
\hline Todas $-\mathrm{Geral}$ & Henricksen & $-17,442^{*}$ & $11,2782^{*}$ & 70,37 & 16,78 & $1,63 \mathrm{E}-16$ & 11,96 & 16,69 \\
$(10$ até $\geq 50 \mathrm{~cm})$ & Stoffels & $1,1638^{*}$ & $0,5367^{*}$ & 70,29 & 15,73 & 1,18 & 11,82 & 16,67 \\
\hline
\end{tabular}


É possível notar que ao realizar o ajuste por classes houve tendência de redução do erro em relação ao ajuste geral, para as classes de regeneração e corte. No entanto, para a classe destinada a corte, observou-se uma redução do coeficiente de determinação ajustado, mostrando que as variações da altura total são pouco explicadas pelas variações do diâmetro a altura do peito. Já para a classe das remanescentes, apesar da redução acentuada do coeficiente de determinação ajustado, houve um aumento do erro. Os valores das estatísticas V, MD e DPD, reforçados pela análise gráfica dos resíduos (Figura 2) e as estatísticas $R^{2}{ }_{a j}$ e $S_{y x}$, oscilaram entre as classes de aproveitamento em relação ao ajuste com todo o banco de dados (sem estratificação).

\section{Regeneração - Classe 1}
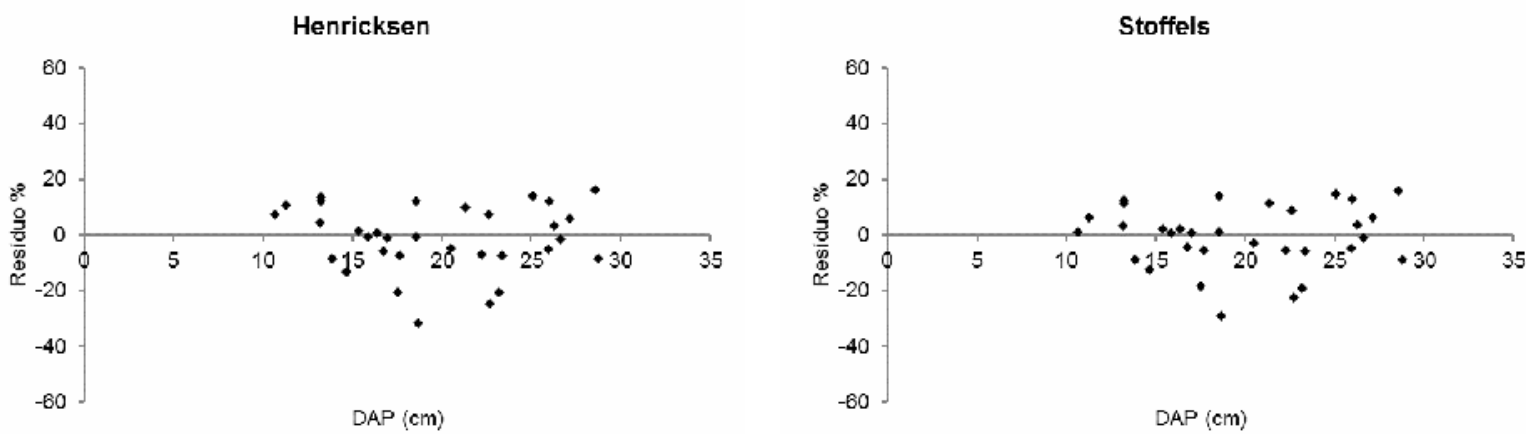

Remanescente - Classe 2
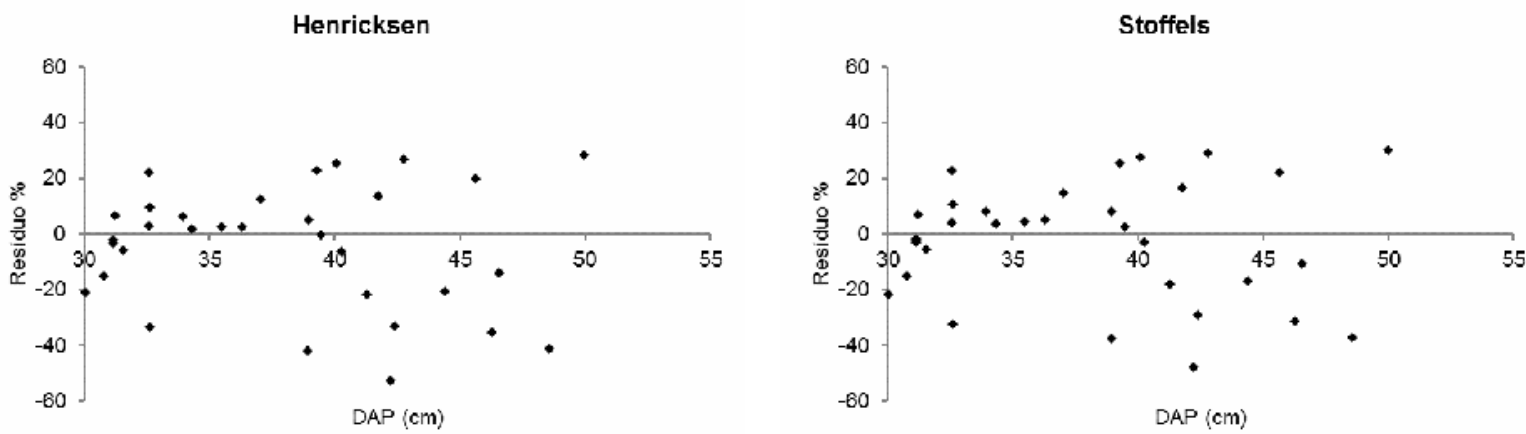

Corte - Classe 3
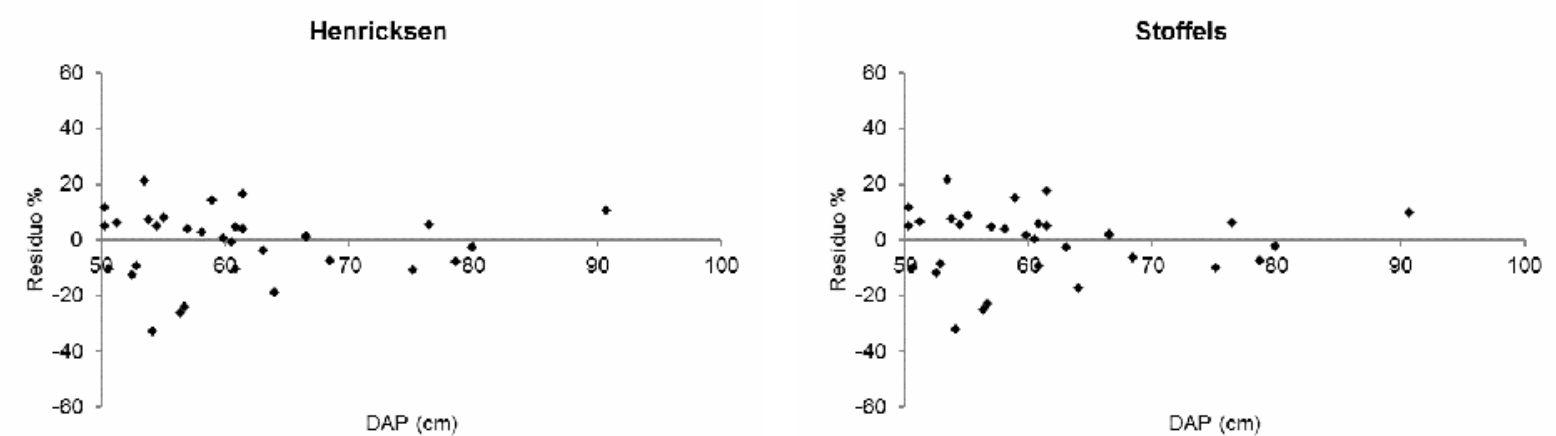

FIGURA 2 - Distribuição dos resíduos de altura, em percentagem, para os modelos de Henricksen e Stoffels, para as classes de regeneração, remanescente e corte, em floresta de transição Cerrado e Amazônia, Sinop, MT. 
Verifica-se que as árvores da classe das remanescentes apresentaram maior dispersão de resíduos. Tal fato pode estar associado ao que foi relatado por Machado et al. (2008), de que em florestas maduras, a competição promove o surgimento de estratos diferentes, ocorrendo indivíduos em posições sociológicas diferenciadas, acarretando variação não proporcional para as variáveis diâmetro e altura.

Ao realizar o ajuste de relações hipsométricas para espécies comerciais (com DAP > $50 \mathrm{~cm}$ ) em floresta Amazônica, Hess et al. (2014) verificaram grande discrepância na relação altura/diâmetro, alegando se tratar de espécies nativas com fustes tortuosos e pouco aproveitáveis, e por ocorrer diâmetros elevados e alturas pequenas ou alturas elevadas com diâmetro menor, gerando assim uma baixa correlação dessas variáveis e valores de $R^{2}{ }_{\text {aj. }}$ não elevados, além do baixo desempenho das demais estatísticas. Confrontando com os dados obtidos no presente trabalho, em que se verificou melhor desempenho da relação hipsométrica para árvores do mesmo porte, é possível notar que no trabalho de Hess et al. (2014) a amplitude dessa classe foi bem mais ampla, variando de 50 até aproximadamente $140 \mathrm{~cm}$, já na área do presente estudo a amplitude foi menor (Figura 2 - Classe de corte), o que pode justificar o melhor desempenho obtido.

Devido a superioridade do modelo de Stoffels após o ajuste de equações, conforme as estatísticas $\mathrm{R}_{\mathrm{aj}}^{2}, \mathrm{~S}_{\mathrm{yx}}, \mathrm{V}, \mathrm{MD}$ e DPD, o teste de identidade entre modelos para as possíveis combinações foi realizado para o referido modelo, conforme apresentado na TABELA 3.

TABELA 3 - Teste de identidade entre modelos hipsométricos

\begin{tabular}{cccc}
\hline Modelo completo & Modelo reduzido & $\mathbf{F}$ & p valor \\
\hline $1+2+3$ & $(123)$ & $67,99^{*}$ & 0,01742 \\
$1+2$ & $(12)$ & $36,29^{\text {ns }}$ & 0,12195 \\
$1+3$ & $(13)$ & $54,14^{*}$ & 0,01978 \\
$2+3$ & $(23)$ & $15,72^{\text {ns }}$ & 0,43101 \\
\hline
\end{tabular}

Em que: * $e^{\text {ns }}$ : significativo e não significativo pelo teste $F$ de Graybill, a 95\% de probabilidade, respectivamente. 1 = Classe $1(10-30 \mathrm{~cm})$ - Regeneração, 2 = Classe $2(30-50 \mathrm{~cm})$ - Remanescente, $3=$ Classe 3 (>50 cm) - Corte.

Com base no teste de identidade entre modelos, foi possível verificar a distinção entre a relação diâmetro-altura entre as classes de aproveitamento avaliadas. No entanto, é possível ajustar uma única equação para descrever a relação hipsométrica das árvores quando se agrupa as classes em regeneração e remanescentes, ou quando se agrupar as árvores remanescentes e corte. Porém, não é possível utilizar a mesma equação para descrever a relação hipsométrica agrupando as árvores em regeneração com as árvores destinadas a corte. Tal resultado realça a importância de se ajustar equações distintas por classe de aproveitamento, para se obter estimativas mais exatas da altura das árvores na floresta. Isso reforça o posicionamento discutido por Gomes et al. (2018), que afirma a necessidade de se utilizar equações específicas para áreas de manejo florestal, uma vez que a estimativa por uma equação geral pode acarretar uma maior amplitude de erro.

Curto et al. (2014) e Trautenmuller et al. (2014) ao realizarem a estratificação de árvores em classes de diâmetro, em uma Floresta Estacional Semidecidual e Floresta Estacional Decidual respectivamente, verificaram que as estimativas de altura total, não 
diferiram estatisticamente daquelas obtidas sem o uso de estratificação. Trautenmuller et al. (2014) verificaram ainda que a estratificação não proporcionou ganhos em precisão e qualidade nos ajustes das equações hipsométricas. Já Curto et al. (2014) notaram maior precisão quando o ajuste foi estratificado por classe de DAP, porém o aumento de precisão foi mais acentuado quando a estratificação foi realizada por espécie. A estratificação de dados por espécies também resultou em melhoria da relação diâmetro e altura em um fragmento de Floresta Ombrófila Mista no Sul do Paraná (SANQUETTA et al. 2013), porém os autores não realizaram teste para verificar ajustes por classes de diâmetro.

Tonini e Borges (2015) recomendam ajustar equações de volume para diferentes espécies, regiões e tipologias florestais, como uma forma indispensável para o planejamento e a execução de um plano de manejo florestal. Do mesmo modo, pode-se sugerir para ajuste de relação hipsométrica que se realize tais estratificações.

Com base nos resultados, verifica-se que a estratificação por classes de aproveitamento proporcionou ganhos em precisão e qualidade nos ajustes apenas para as classes de regeneração e corte (TABELA 5), e que há possibilidade de agrupar as classes regeneração com remanescente e remanescente com corte (TABELA 6). Assim, conforme recomendado por Curto et al. (2014) a utilização das estratificações deverá ser decidida pelo pesquisador de acordo com os objetivos de sua pesquisa, já que estas podem influenciar no tempo de coleta e no processamento dos dados, assim como nos custos dos mesmos.

\section{CONCLUSÕES}

Equações hipsométricas estratificadas por classe de aproveitamento resultam em aumento da precisão quando trata-se de árvores nas classes de regeneração (10 a $29,9 \mathrm{~cm}$ de DAP) e de corte $(>50 \mathrm{~cm}$ ).

Quanto a identidade de modelos, é possível o agrupamento de árvores nas classes regeneração com as remanescentes, e agrupamento de árvores remanescentes com árvores da classe de corte, para realização simultânea dos ajustes. Pela estratificação e o aumento de precisão, pode-se reduzir tempo de coleta de dados e custos envolvidos na atividade, com a redução da intensidade amostral.

\section{REFERÊNCIAS}

ARAÚJO, E. J. G.; PELISSARI, A. L.; DAVID, H. C.; SCOLFORO, J. R. S.; PÉLLICO NETTO, S.; MORAIS, V. A. Relação hipsométrica para candeia (Eremanthus erythropappus) com diferentes espaçamentos de plantio em Minas Gerais, Brasil. Pesquisa Florestal Brasileira, v. 32, n. 71, p. 257-268, 2012. DOI: ttps://doi.org/10.4336/2012.pfb.32.71.257

ARAÚJO, R. A.; COSTA, R. B.; FELFILI, J. M.; KUNTZ, I. G.; SOUZA, R. A. T. de M. e; DORVAL, A. Florística e estrutura de fragmento florestal em área de transição na Amazônia Matogrossense no município de Sinop. Acta Amazônica, Manaus, AM, v.39, n.4, p.865-877, 2009. DOI: 10.1590/S0044-59672009000400015

BRASIL. Ministério de Meio Ambiente. Resolução Conama n $^{\circ} 406$, de 02 de fevereiro de 2009. Estabelece parâmetros técnicos a serem adotados na elaboração, 
apresentação, avaliação técnica e execução de Plano de Manejo Florestal SustentávelPMFS com fins madeireiros, para florestas nativas e suas formas de sucessão no bioma Amazônia. Diário Oficial da República Federativa do Brasil, № 26, Brasília, DF, 2009.

BRASIL. Ministério de Meio Ambiente. Instrução normativa no 5, de 11 de dezembro de 2006. Dispõe sobre procedimentos técnicos para elaboração, apresentação, execução e avaliação técnica de Planos de Manejo Florestal Sustentável-PMFSs nas florestas primitivas e suas formas de sucessão na Amazônia Legal, e dá outras providências. Diário Oficial da República Federativa do Brasil, Brasília, DF, 2006.

CURTO, R. A.; MÔRA, R.; ARAÚJO, E. J. G.; SILVA, G. F. Treinamento na estimativa da altura de árvores de grande porte em floresta inequiânea. Scientia Agraria Paranaensis, Marechal Cândido Rondon, v. 17, n. 2, p. 170-176, 2018.

CURTO, R. A.; LOUREIRO, G. H.; MÔRA, R.; MIRANDA, R. O. V.; PÉLLICO NETTO, S. SILVA, G. F. Relações hipsométricas em Floresta Estacional Semidecidual. Revista de Ciências Agrárias, v. 57, n. 1, p. 57-66, 2014. DOI: http://dx.doi.org/10.4322/rca.2013.066

CURTO, R. A.; SILVA, G. F.; SOARES, C. P. B.; MARTINS, L. T.; DAVID, H. C. Métodos de estimação de altura de árvores em Floresta Estacional Semidecidual. Revista Floresta, Curitiba, PR, v.43, n.1, p.105-116, 2013. DOI: 10.5380/rf.v43i1.26791

FELICIANO, M. E.; RIBEIRO, A.; FERRAZ FILHO, A. C.; VITOR, P. C. G. Avaliação de diferentes hipsômetros na estimativa da altura total. Revista Verde de Agroecologia e $\begin{array}{lllll}\text { Desenvolvimento } \quad \text { Sustentável, } & \text { v.11, } & \text { n.2, } & \text { p.01-05, }\end{array}$ DOI: https://doi.org/10.18378/rvads.v11i2.3741

FIGUEIREDO FILHO, A; BORDERS, B. E.; HITCH K. L. Taper equations for Pinus taeda plantations in Southern Brazil. Forest Ecology and Management. v. 83, p. 3946, 1996.

GAMA, J. R. V.; SOUZA, A. L.; VIEIRA, D. S.; LEITE, H. G. Equações de volume para uma Floresta Ombrófila Aberta, município de Codó, estado do Maranhão. Revista Brasileira de Ciências Agrárias, v. 12, n. 4, p. 535-542, 2017. DOI: 10.5039/agraria.v12i4a5489

GIMENEZ, B. O.; DANIELLI, F. E.; OLIVEIRA, C. K. A.; SANTOS, J.; HIGUCHI, N. Equações volumétricas para espécies comerciais madeireiras do sul do estado de Roraima. Scientia Forestalis, v. 43, n. 106, p. 291-301, 2015.

GOMES, K. M. A.; SILVA-RIBEIRO, R. B.; GAMA, J. R. V.; ANDRADE, D. F. C. Eficiência na estimativa volumétrica de madeira na Floresta Nacional do Tapajós. $\begin{array}{lllllll}\text { Revista Nativa, } & \text { v. } 6, \quad \text { n. } & 2, & \text { p. } & 170-176,\end{array}$ DOI: http://dx.doi.org/10.31413/nativa.v6i2.5237 
GRAYBILL, F. A. Theory and Application of the Linear Model. Belmont: Duxbury Press, 1976. 704p.

HESS, A. F.; BRAZ, E. M.; THAINES, F., MATTOS, P. P. Ajuste de relação hipsométrica para espécies da Floresta Amazônica. Ambiência, v. 10, n. 1, p. 21-29, 2014. DOI:10.5935/ambiencia.2014.01.02

LEVINE, D. M.; STEPHAN, D. F.; SZABAT, K. A. Estatística: Teoria e Aplicações. 7aㅗ Ed. Rio de Janeiro, RJ: Livros Técnicos e Científicos, 2017. 760 p.

LINGNAU, C., SILVA, M. N., SANTOS, D.S, MACHADO, A., LIMA, J. G. S. Mensuração de alturas de árvores individuais a partir de dados laser terrestre. Ambiência, Guarapuava, PR, v.4, Edição Especial, p.85-96, 2008.

MACHADO, S. A.; NASCIMENTO, R. G. M.; AUGUSTYNCZIK, A. L. D.; SILVA, L. C. R.; FIGURA, M. A.; PEREIRA, E. M.; TÉO, S. J. Comportamento da relação hipsométrica de Araucaria angustifolia no capão da Engenharia Florestal da UFPR. Pesquisa Florestal Brasileira, n. 56, p. 5-16, 2008.

MACHADO, S. A.; ACCIOLY, Y.; NASCIMENTO, R. G. M.; SILVA, L. C. R.; CARDOZO, C. C. Influência do comprimento de copa na relação hipsométrica de Araucaria angustifolia. Pesquisa florestal brasileira, Colombo, v. 35, n. 83, p. 343-351, 2015. DOI: https://doi.org/10.4336/2015.pfb.35.83.493

Mato Grosso. Secretaria de Estado do Meio Ambiente. Instrução Normativa no 005 , de 02 de julho de 2014. Estabelece roteiros específicos para Plano de Manejo Florestal Sustentável-PMFS Madeireiro. Diário Oficial do Estado de Mato Grosso, Cuiabá, MT, 2014.

MATO GROSSO. Secretaria de Estado do Meio Ambiente. Decreto № 1.862, de 24 de março de 2009. Regulamenta a Lei Complementar no 233, de 21 de dezembro de 2005 no que diz respeito aos procedimentos de elaboração, análise e acompanhamento dos Planos de Manejo Florestal Sustentável no Estado de Mato Grosso. Diário Oficial do Estado de Mato Grosso, Cuiabá, MT, 2009.

MOTA L. L.; BOTON D.; FONSECA, R. C.; SILVA W. C.; SOUZA A. P.; Balanço hídrico climatológico e classificação climática da região de Sinop, Mato Grosso. Scientific Eletronic Archives, v.3, p.38-44, 2013.

REGAZZI, A. J. Teste para verificar a identidade de modelos de regressão. Pesquisa Agropecuária Brasileira, Brasília, v. 31, n. 1, p. 1-17, 1996.

SANQUETTA, C. R.; DAllA CORTE, A. P.; ROGLIN, A.; PIMENTEL, A. Relações diâmetro-altura para espécies lenhosas em um fragmento de Floresta Ombrófila Mista no Sul do Paraná. Iheringia, Sér. Bot., Porto Alegre, v. 68, n. 1, p. 103-114, 2013. 
SILVA, G. F. da; CURTO, R. de A.; SOARES, C. P. B.; PIASSI, L. de C. Avaliação de métodos de medição de altura em florestas naturais. Revista Árvore, Viçosa, MG, v.36, n.2, 2012. DOI: http://dx.doi.org/10.1590/S0100-67622012000200015.

SILVA, G. F.; MÔRA, R.; CURTO, R. de A. Simulação de erros na medição de altura de árvores inclinadas com aparelhos baseados em princípios trigonométricos. Revista Nativa, Sinop, MT, v.5, n.5, p. 372-379, 2017. DOI: http://dx.doi.org/10.5935/23187670.v05n05a12

SOUZA, A. L.; SOUZA D. R. Estratificação vertical em floresta ombrófila densa de terra firme não explorada, Amazônia Oriental. Revista Árvore, v.28, n.5, p.691-698, 2004. DOI: $10.1590 / S 0100-67622004000500008$

TRAUTENMÜLLER, J. W.; BALBINOT, R.; GONZATTO, G. L.; WATZLAWICK, L. F.; VENDRUSCOLO, R. Relação hipsométrica em Floresta Estacional Decidual. Enciclopédia biosfera, Centro Científico Conhecer - Goiânia, v.10, n.19, 2014.

TONINI, H.; BORGES, R. A. Equação de volume para espécies comerciais em Floresta Ombrófila Densa no sul de Roraima. Pesquisa Florestal Brasileira, v.35, n.82, p.111117, 2015. Doi: 10.4336/2015.pfb.35.82.738

VILLASANTE, A.; FERNANDEZ, C. Measurement errors in the use of smartphones as low-cost forestry hypsometers. Silva Fennica, v. 48, n.5, 11 p., 2014. DOI: $10.14214 /$ sf. 1114

SANTOS, M. J. F.; ANDRADE, V. C. L.; SOUZA, P. B. Amostragem relativa de dados da relação hipsométrica de Cerrado Tocantinense. Revista Brasileira de Biometria., v. 34, n. 4, p. 633-645, 2016.

STAUB, C. M. M.; SANQUETTA, C. R.; DALLA CORTE, A. P.; SANQUETTA, M. N. I.; VASCONCELLOS, B. N. Equações hipsométricas para plantios mistos de restauração florestal na Mata Atlântica em Seropédica-RJ. Enciclopédia biosfera, Centro Científico Conhecer - Goiânia, v.10, n.18, 2014. 